\title{
PARP inhibitors for small cell lung cancer and their potential for integration into current treatment approaches
}

\author{
Ranya Barayan ${ }^{1 \#}$, Xiaozhuo Ran ${ }^{2 \#}$, Benjamin H. Lok ${ }^{1,2,3,4}$ \\ ${ }^{1}$ Institute of Medical Science, Faculty of Medicine, University of Toronto, ON, Canada; ${ }^{2}$ Radiation Medicine Program, Princess Margaret Cancer \\ Centre, Toronto, ON, Canada; ${ }^{3}$ Department of Medical Biophysics, ${ }^{4}$ Department of Radiation Oncology, University of Toronto, Toronto, ON, \\ Canada \\ Contributions: (I) Conception and design: All authors; (II) Administrative support: None; (III) Provision of study materials or patients: None; (IV) \\ Collection and assembly of data: None; (V) Data analysis and interpretation: None; (VI) Manuscript writing: All authors; (VII) Final approval of \\ manuscript: All authors. \\ "These authors contributed equally to this work. \\ Correspondence to: Benjamin H. Lok, MD, FRCPC. Clinician Scientist and Staff Radiation Oncologist at the Princess Margaret Cancer Centre, \\ University Health Network. 610 University Ave, Toronto, ON, M5G 2M9, Canada. Email: Benjamin.Lok@rmp.uhn.ca.
}

\begin{abstract}
Small cell lung cancer (SCLC) is a very aggressive, highly lethal, neuroendocrine tumor that constitutes $15 \%$ of all lung cancer cases. It is characterized by its rapid disease progression and high relapse rate leading to poor survival for diagnosed patients. Recently, poly (ADP-ribose) polymerase inhibitors (PARPi) have emerged as a novel therapeutic strategy for SCLC. Preclinical studies have demonstrated that PARPi possesses cytotoxic activity as a single-agent and in combination with other anti-cancer agents. Predictive biomarkers of response to PARPi, such as SLFN11, have also been described in SCLC. This review aims to summarize the recent preclinical investigations and the relevant clinical trials that evaluate PARPi in SCLC. Here, we highlight the potential role of PARPi in a biomarker-selected manner and in combination with chemotherapy, targeted agents, radiotherapy and immunotherapy.
\end{abstract}

Keywords: Small cell lung cancer (SCLC); poly (ADP-ribose) polymerase inhibitors (PARPi); chemotherapy; radiation sensitizer; immunotherapy

Submitted Mar 03, 2020. Accepted for publication Mar 16, 2020.

doi: $10.21037 /$ jtd.2020.03.89

View this article at: http://dx.doi.org/10.21037/jtd.2020.03.89

\section{Introduction}

Lung cancer has remained the most common cancer worldwide since 1985, with approximately 1.8 million new cases diagnosed each year $(1,2)$. Among the major lung cancer subtypes, small cell lung cancer (SCLC) represents the sixth leading cause of cancer-related mortality, accounting for $13-15 \%$ of all lung cases $(3,4)$. Clinically, SCLC is considered an aggressive and lethal highgrade neuroendocrine malignancy that is pathologically, molecularly, and biologically very distinct from other forms of lung cancer. Almost all SCLC cases have homozygous loss of $R B 1$, which encodes the key regulator of the G1-S cell cycle checkpoint, and TP53, a gene critical for multiple
DNA damage response (DDR) pathways (5-11). This can, in part, explain the initial responsiveness of SCLC to various DNA damaging agents, such as those that induce covalent DNA adducts and crosslinks [cisplatin, carboplatin, temozolomide (TMZ)], or those that produce single-strand/ double-strand DNA breaks (ionizing radiation, etoposide, topotecan, irinotecan).

The standard of care for first-line treatment of SCLC consists of a platinum-based doublet chemotherapy regimen for all patients, concurrent with radiotherapy (RT) for those with limited-stage disease. Despite a high (70-80\%) initial response rate to these first-line regimens, the 5 -year overall survival (OS) rates for patients diagnosed with SCLC is a dismal $6.5 \%$ (12). Until 2019, with the exception 
of the FDA approval of atezolizumab with chemotherapy for extensive-stage SCLC (ES-SCLC) patients, the general treatment paradigm has remained unchanged for the previous several decades $(13,14)$. Therefore, novel therapeutic interventions are needed and are an active area of research (15-17).

One such novel therapeutic are inhibitors of poly-(ADP)ribose polymerase (PARP), which have demonstrated efficacy against SCLC in preclinical and clinical data over the past several years. Poly (ADP-ribose) polymerase inhibitors (PARPi) have the potential to enhance cytotoxic response to chemotherapy, radiotherapy, and immunotherapy in SCLC. This review will highlight the advances made in these areas.

\section{Current application of PARPi in oncology}

PARP is a family of proteins that orchestrate various cellular processes and have important roles in DNA repair and genome integrity. PARP1 activates base excision repair (BER) in response to DNA single-strand breaks (SSBs) where PARP1 binds to SSBs and facilitates the recruitment of DNA repair proteins. When PARP1 function is impaired, the BER process is halted and double-strand breaks (DSBs) develop due to a destabilized replication fork (18). As a result, malignancies deficient in the DSB repair pathway of homologous recombination (HR) are vulnerable to PARP inhibition. PARPi were first demonstrated to have efficacy in ovarian cancers with BRCA1/2 mutations-which are deficient in HR (19). Subsequently, PARPi clinical efficacy has extended to other histologies harboring BRCA1/2 mutations (19-27) with most PARPi carrying FDA approval for treating $B R C A 1 / 2$-mutated ovarian and breast cancers (Table 1) (30-37).

The various PARPi generally have similar activity in their degree of polymerase inhibition. However, the PARPi differ in their ability to poison and trap PARP to the DNA SSB lesions which consequently devolve into cytotoxic DSBs upon DNA replication (38). Talazoparib has been demonstrated to be the most potent PARP trapper followed by niraparib, then olaparib and rucaparib, with veliparib being the least potent trapper $(28,29)$. These differences between their potency in PARP trapping may inform their observed efficacy and side effect profiles.

Recently, studies have examined the utility of PARPi beyond BRCA1/2-mutant tumors (39). Several groups have demonstrated that large chromosomal structural alterations, characteristic of these $B R C A 1 / 2$-mutant cancers, can be quantitated by three correlated HR deficiency (HRD) metrics: loss of heterozygosity $(\mathrm{LOH})$, large-scale state transition (LST), and telomeric allelic imbalance $\left(\mathrm{N}_{\mathrm{tAI}}\right)$ (40-43). These HRD scores correlate with sensitivity to platinum agents of sporadic triple-negative breast and ovarian cancers $(40-42,44,45)$. This suggests that PARPi may have therapeutic benefits in any malignancy harboring HRD independent of the canonical BRCA1/2 mutations. As $B R C A$ mutations are rare $(\leq 2 \%)$ in SCLC, this concept of non-BRCA dependent PARPi sensitivity lay the foundation for subsequent investigations $(10,11)$.

\section{PARP as a therapeutic target in SCLC}

\section{Pre-clinical evidence and studies}

\section{Rationale for PARPi in SCLC}

In 2012, Byers et al. conducted a landmark study where reverse phase protein array (RPPA) for proteomic analysis of 34 SCLC and 74 non-SCLC (NSCLC) cell lines identified potential targets unique to SCLC. The analysis revealed that SCLC cell lines had high PARP1 protein expression relative to NSCLC. In addition, SCLC patient tumors demonstrated high PARP1 protein expression when compared to other neuroendocrine tumors and NSCLC. In vitro cell line drug response studies with olaparib and rucaparib confirmed that most SCLC cell lines tested were highly sensitive to treatment with PARPi in contrast to NSCLC cell lines (46). However, PARPi drug sensitivity was not universal for all SCLC cell lines tested. Therefore, interest towards identifying molecular mechanisms of sensitivity as potential predictive biomarkers increased.

\section{Potential predictive biomarkers of PARPi response}

Scblafen family member 11 (SLFN11) was recently identified as a putative predictive biomarker for SCLC sensitivity to PARPi. Multiple independent groups demonstrated that high SLFN11 gene or protein expression levels positively correlate with increased PARPi treatment sensitivity (47-51).

Polley et al. examined 63 SCLC cell-lines in response to treatment with multiple PARPi (talazoparib, olaparib, niraparib, rucaparib, AZD-2461) in their screen of 103 FDA approved oncology drugs and 423 investigational agents (47). Results indicated that increased gene expression of $S L F N 11$ correlated with decreased $\mathrm{IC}_{50}$ (i.e., inhibitory concentration producing $50 \%$ growth inhibition) values to all tested PARPi $(\mathrm{R}=-0.42)$. Of note, expression levels of neither PARP1 nor PARP2 had any predictive value (47). 


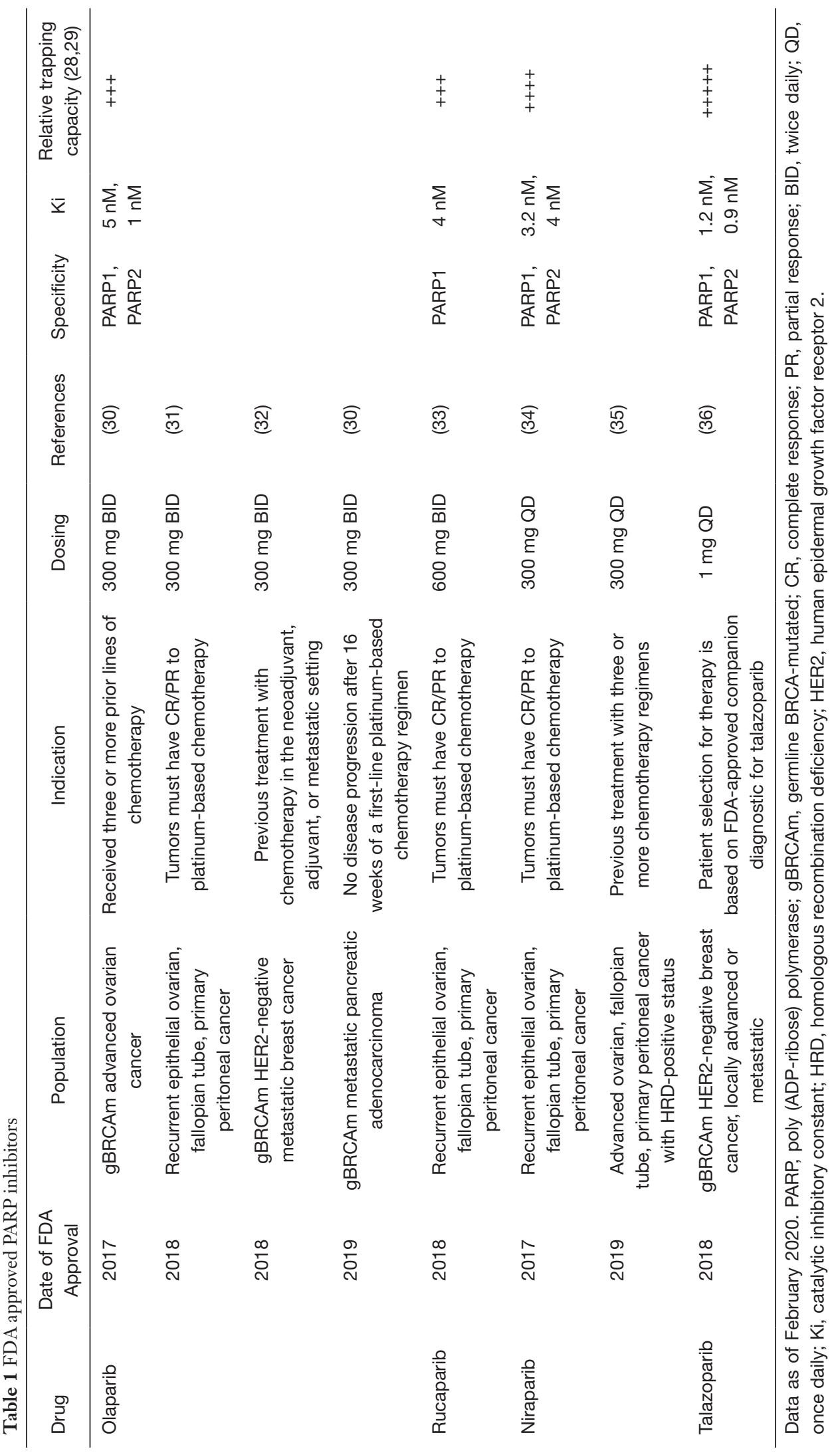


Similarly, a significant correlation between SLFN11 expression levels and response to talazoparib was identified in a study by Murai et al. (48). Importantly, the relationship between SLFN11 expression and PARPi sensitivity was determined to be causal, where CRISPR-mediated genetic knockout of SLFN11 in 4 cell lines with high SLFN11 (prostate DU145, leukemia CCRF-CEM and MOLT4, and Ewing's sarcoma EW8) resulted in resistance to both talazoparib and olaparib relative to their parental cell lines. Further confirmation was achieved when exogenous expression of SLFN11 was induced in leukemia K562 cells (that have low SLFN11 endogenous transcript) and resulted in hypersensitivity to both talazoparib and olaparib. Murai et al. concluded that SLFN11 is a dominant determinant of PARPi sensitivity in these cancer cells (48).

Specific to SCLC, Lok et al. observed that SLFN11 gene and protein expression levels correlated to PARPi response in SCLC cell lines and patient-derived xenograft (PDX) mouse models (49). The study showed that high expression of SLFN11 positively correlated with increased sensitivity to various PARPi (i.e., olaparib, rucaparib, veliparib, and talazoparib) in cell line datasets. Functionally, genetic knockdown and knockout of SLFN11 in SCLC cell lines conferred resistance to PARPi. Immunohistochemical (IHC) staining of SLFN11 confirmed these findings in multiple PDX models treated with talazoparib (49). These studies suggested that $S L F N 11$ could be used as a predictive biomarker of response to PARPi monotherapy in SCLC.

Stewart et al. reported that there may be two potential biomarkers (SLFN11 and ATM) with predictive capability of PARPi response in SCLC. In this study, 170 proteins quantified by RPPA were investigated as potential predictive biomarkers in response to single-agent treatment with talazoparib. Results revealed that low ATM and high SLFN11 protein expression were significantly associated with treatment response in SCLC PDX models. While high CHK1, IGF1R beta, and IRS1 protein levels correlated with resistance. These findings were validated at the mRNA level which showed the strongest association between talazoparib response and high SLFN11, low ATM, and low CHEK1 expression in SCLC PDX models (50).

Multiple laboratories have investigated the mechanisms by which SLFN11 may confer PARPi sensitization (52-54). Mu et al. reported the interaction of SLFN11 with replication protein A (RPA) led to the suppression of HR. SLFN11 destabilized RPA1-ssDNA complexes necessary for efficient downstream DNA repair by HR (52). Murai et al. observed that SLFN11 was recruited to DNA damage sites where it binds with RPA and subsequently to the minichromosome maintenance protein complex (MCM) DNA helicase that is essential for DNA replication. Although SLFN11 did not directly engage in replication initiation, SLFN11 unwound heterochromatin, blocked the progression of the replication fork, and ultimately hindered the DNA repair process (53). Li et al. reported indirect inhibition of ATM and ATR protein synthesis by SLFN11. The absence of these DNA repair proteins promoted sensitization to the effects of DNA damaging agents (54). Continued investigation into the contribution of these mechanisms to PARPi sensitivity would be of considerable basic and translational interest as these therapeutics are being actively investigated in multiple clinical trials of several histologies, including SCLC.

\section{Clinical evidence and studies}

\section{Single-agent PARPi trials}

In a phase I trial (NCT01286987), 113 patients with recurrent breast, ovarian, prostate, and pancreatic cancers containing deficiencies in DNA repair pathways were recruited, in addition to 23 relapsed SCLC patients. This study was designed to assess the safety and efficacy of talazoparib monotherapy. The dose escalation cohort of this study evaluated 39 patients, none of which were SCLC, and determined the maximum tolerated dose (MTD) to be $1.0 \mathrm{mg}$ of talazoparib daily. The subsequent cohort of this study assessed 71 patients, including all 23 SCLC patients, who were administered talazoparib $1.0 \mathrm{mg}$ daily. Talazoparib demonstrated the highest bioavailability and anti-tumor activity in patients presenting with $B R C A$ mutations [objective response rate (ORR) $>40 \%$ ] (55). Of the 23 SCLC patients, 2 patients had a partial response (ORR $=9 \%)$ that lasted between 3-4 months. Both patients previously had an objective response to prior platinum therapy and were platinum-free for up to 6 months. The median progression-free survival (PFS) of these patients was 11.1 weeks [95\% confidence interval (CI): 4.3-13.0 weeks]. An additional 4 SCLC patients had stable disease (SD) that lasted at least 16 weeks [clinical benefit rate (CBR), $26 \%>16$ weeks] (55). These data suggest that incorporating a predictive biomarker to select SCLC patients that may benefit from PARPi monotherapy would be prudent.

As such, there is an ongoing biomarker-selected PARPi monotherapy phase II clinical trial (NCT03009682), investigating the efficacy of olaparib in relapsed SCLC patients whose tumors harbor mutations in HR related 
genes including $B R C A 1 / 2, A T M, B L M, M R E 11, R A D 50$, $N B N, R A D 51, R A D 51$ paralogs, RECQL family members, and other deleterious HR pathway alterations. Results from this biomarker informed clinical trial are eagerly awaited (summarized in Table 2).

Given that the benefit of single-agent PARPi for SCLC therapy may have limited efficacy outside of biomarkerselected patients, combining PARPi with other therapeutics is a rational next step. In subsequent sections, we will review the landscape of PARPi combination studies with chemotherapy, targeted therapeutics, radiotherapy and immunotherapy.

\section{PARPi and drug combinations in SCLC}

Chemotherapy remains the standard of care for treating SCLC patients. ES-SCLC patients treated with chemotherapy have a low median survival of about 10 months $(63,64)$ with immunotherapy increasing that to about 12 months $(65,66)$. There is a critical need to improve treatment efficacy and outcomes for SCLC patients that PARPi may be able to contribute toward. Several preclinical studies demonstrated that PARPi chemosensitizes SCLC. These data informed the subsequent development of related clinical trials.

\section{Preclinical evidence and studies}

Byers et al. first reported that the addition of olaparib to the standard platinum-based chemotherapy regimen, cisplatin and etoposide (CE), potentiated the anti-tumor effects in SCLC (46). Owonikoko et al. similarly reported synergism between veliparib combined with a platinum-based agent (cisplatin/carboplatin) and etoposide in SCLC cell lines as well as xenograft mouse models (67). Teicher et al. demonstrated enhanced sensitivity to carboplatin/etoposide treatment with talazoparib in some SCLC cell lines (68). Several preclinical reports indicated that PARPi can also sensitize SCLC to other chemotherapeutic agents. For example, Murai et al. observed that talazoparib sensitizes cancer cells to the DNA-alkylating agent TMZ, and that sensitization was dependent on SLFN11 expression (48). Lok et al. also found evidence of synergy to the combination of talazoparib and TMZ (49).

Beyond PARPi combinations with chemotherapy, multiple groups have examined combining novel targeted therapies with PARPi (69-71). Lallo et al. showed that the combination of PARP inhibitor olaparib and WEE1 inhibitor adavosertib (AZD1775) can significantly improve the efficacy of the single-agent activity of olaparib in SCLC circulating tumor cell patient-derived xenografts (CDX) (69). A study in abstract form by Gay et al. also demonstrated synergism between an ATR inhibitor (AZD6738) and olaparib that increased the cytotoxic effects in SCLC cell lines (70). While Sen et al. indicated that a CHK1 inhibitor (LY2606368) synergizes with olaparib to decrease cell viability and cause tumor regression in a tripleknockout $\mathrm{RB}^{-/} / \mathrm{p} 53^{-/-} / \mathrm{p} 130^{-/-}$(RPP) genetically engineered mouse model (GEMM) (71). These studies collectively demonstrated the potential of a combinatorial approach as an effective therapeutic strategy for the incorporation of PARPi into the management of SCLC.

\section{Clinical evidence and studies}

PARPi combination trials with cisplatin and etoposide The combination of chemotherapy and PARPi has been studied in clinical trials. Two independent studies evaluated the feasibility of veliparib in combination with $\mathrm{CE}$ as a therapeutic strategy in SCLC $(59,60)$. Owonikoko et al. completed a phase I/II clinical trial (NCT01642251) to evaluate the safety and efficacy of veliparib combined with CE in SCLC patients. A total of 128 ES-SCLC patients were recruited and treated with four cycles of $\mathrm{CE}$ along with veliparib or placebo. The toxicities were balanced between the two treatment groups with the exception of higher grade $3+$ lymphopenia ( $8 \%$ vs. $0 \% ; \mathrm{P}=0.06$ ) and neutropenia ( $49 \%$ vs. $32 \% ; \mathrm{P}=0.08$ ) in the veliparib group compared to placebo. The primary endpoint of the study was to examine if the veliparib combination would reduce the PFS hazard ratio by $37.5 \%$ as analysed by a one-sided log rank test with an $\alpha$ of 0.10 . Results demonstrated a higher median PFS (6.1 vs. 5.5 months; one-sided $\mathrm{P}$ value $=0.06$ ) and median $\mathrm{OS}$ (10.3 vs. 8.9 months; $\mathrm{P}=0.17$ ) in the veliparib group, which indicated that the addition of veliparib may improve current CE treatment (59). In another phase I dose-escalation study (NCT 02289690), Atrafi et al. reported that 16 of the 25 ES-SCLC patients (64\%) had confirmed responses to the combination of veliparib and carboplatin/etoposide with this proportion increasing to $83 \%$ (5 of 6) of SCLC patients treated at the recommended phase II dose of veliparib. To date, the phase II portion of this study is ongoing (60).

\section{PARPi combination trials with TMZ}

Farago et al. reported a phase I/II clinical trial (NCT02446704), where the phase II of this study 


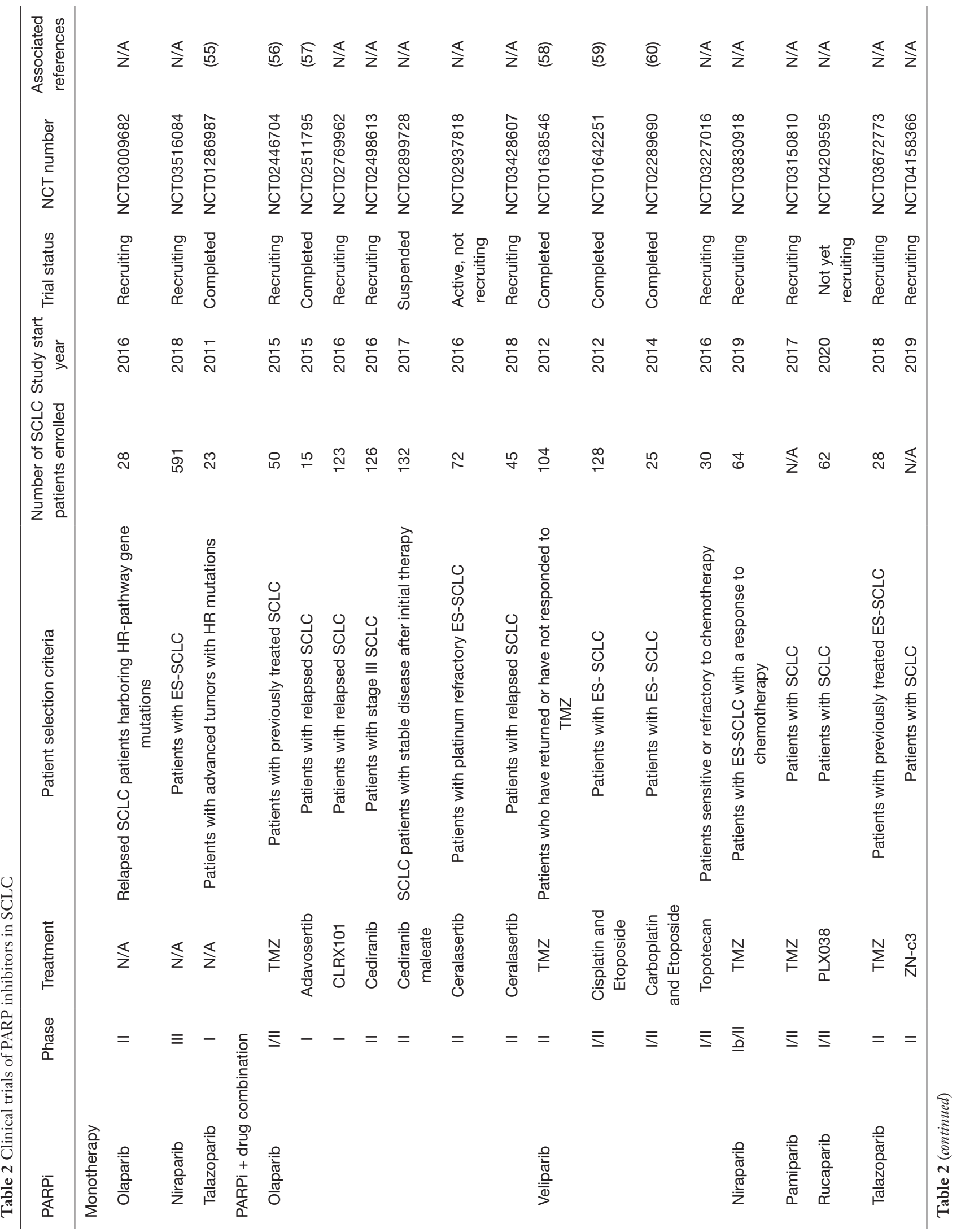




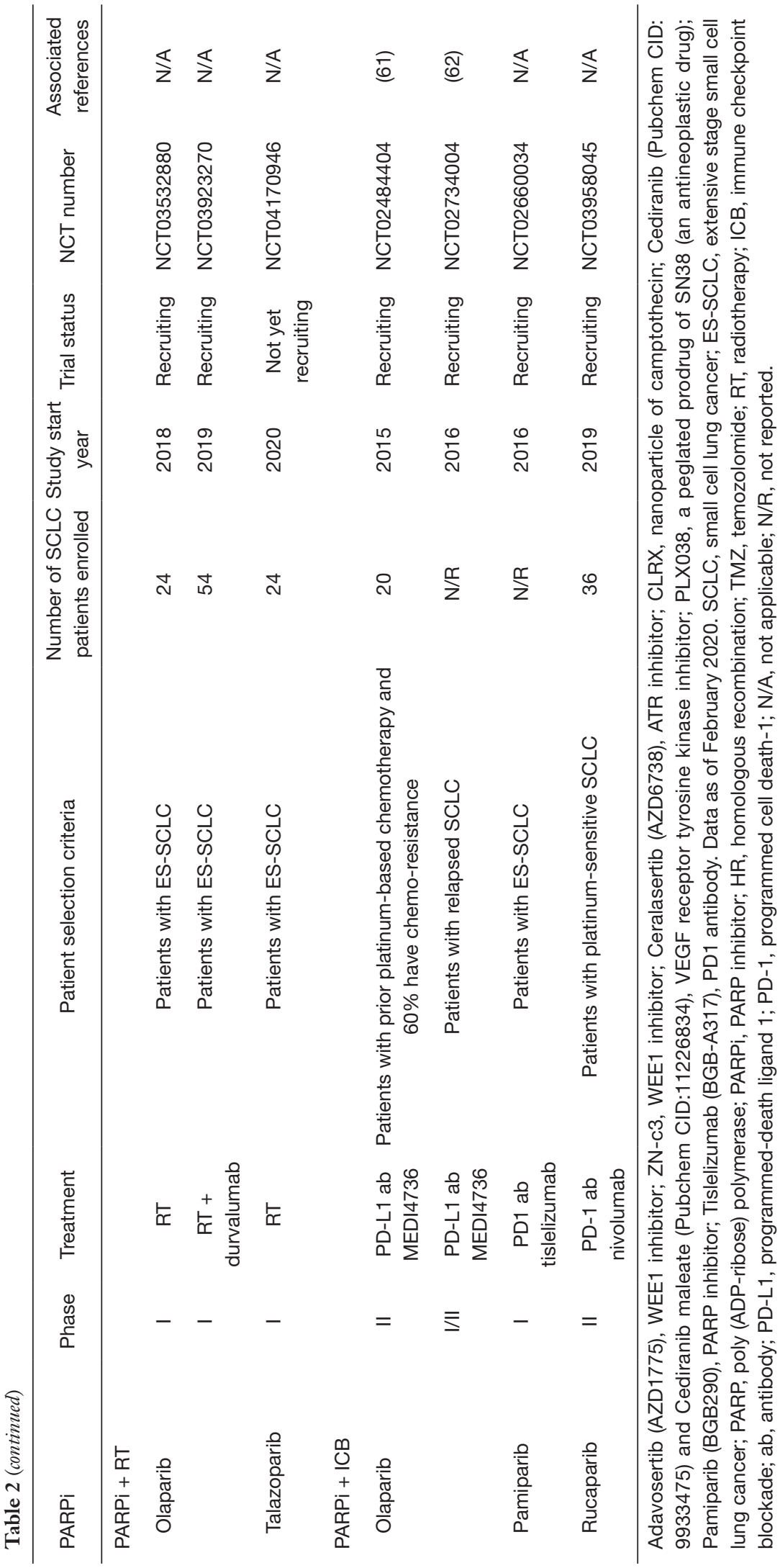


consisted of 50 patients that were administered $200 \mathrm{mg}$ of olaparib orally twice daily combined with $75 \mathrm{mg} / \mathrm{m}^{2}$ of TMZ daily on days 1-7 per 21 days. Results from this study showed an ORR of $41.7 \%$, PFS of 4.2 months, and OS of 8.5 months (56).

Another phase II, randomized, double-blinded clinical study conducted by Pietanza et al. (NCT01638546) also showed the promise of PARPi in SCLC therapy. In their study, 104 patients with recurrent SCLC were recruited and treated with either TMZ $150-200 \mathrm{mg} / \mathrm{m}^{2}$ on days $1-5$ per 28 days in combination with veliparib $40 \mathrm{mg}$ twice daily or placebo for days 1-7 per 28 days. Although the results showed no significant differences in 4-month PFS and OS between the two groups, significant differences were observed in the ORR where patients receiving TMZ/ veliparib had a higher ORR than TMZ alone (39\% vs. $14 \% ; \mathrm{P}=0.016)$. Interestingly, in an exploratory analysis, significantly prolonged PFS and OS was observed in the $\mathrm{TMZ} /$ veliparib treated patients with high protein expression of SLFN11 (58).

\section{PARPi combination trials with other drugs}

In addition to combination studies of PARPi with firstline chemotherapeutic agents, clinical trials evaluating the efficacy of other drug combinations with PARPi for SCLC are currently ongoing. In a phase I clinical study (NCT02511795) of 15 relapsed SCLC patients, the relative safety of olaparib in combination with adavosertib is being evaluated (57). Other clinical trials that are ongoing include a phase II study (NCT02498613) of olaparib and cediranib (VEGF inhibitor) in multiple cancer histologies, including SCLC, and a phase II trial (NCT03227016) of veliparib and topotecan in SCLC patients sensitive or refractory to chemotherapy (summarized in Table 2).

\section{PARPi and RT in SCLC}

$\mathrm{RT}$ is an effective treatment strategy for various cancers and has been proven to improve local tumor control and survival rates for limited-stage SCLC patients when combined with chemotherapy $(72,73)$, while reports of the effectiveness of thoracic RT for ES-SCLC have been mixed (74-76). Results from a phase III randomized clinical study by Slotman et al. in 495 ES-SCLC patients reported that consolidative radiation therapy (cRT; $30 \mathrm{~Gy} / 10$ fractions over 2 weeks) to the thorax when added to prophylactic cranial irradiation (PCI) significantly improved the secondary endpoints of
PFS at 6 months (24\% vs. 7\%, $\mathrm{P}=0.001)$ and $\mathrm{OS}$ at 2 years ( $13 \%$ vs. 3\%, $\mathrm{P}=0.004)$ but not its primary endpoint of OS at 1 year $(33 \%$ vs. $28 \%, \mathrm{P}=0.066)(75)$. In another clinical study (RTOG0937), 86 ES-SCLC patients were randomized to PCI alone or to cRT (45 Gy/15 fractions over 3 weeks) to intrathoracic disease and up to 4 extracranial metastases in addition to PCI. This more aggressive RT strategy did not improve OS at 1 year $(60.1 \%$ in PCI alone vs. $50.8 \%$ in PCI + cRT) (76). With these mixed results for the role of RT in ES-SCLC, novel strategies to understand and improve the application of RT for SCLC patients are warranted.

\section{Pre-clinical evidence and studies}

PARP1 plays a crucial role in several DDR pathways, and inhibiting the function of PARP1 perturbs the cell's ability to respond to RT-induced DNA damage. Indeed, Owonikoko et al. showed that SCLC cell lines were significantly sensitized to RT when combined with veliparib (67). PARP trapping also contributed to radiosensitization as documented by Laird et al. who observed that increased radiosensitization of SCLC cell lines and PDXs was most prominent with the more potent PARP trapper, talazoparib, as compared to veliparib, a lower potency PARP trapper (77). In addition to understanding the mechanisms by which radiosensitivity is achieved, pharmacodynamic imaging biomarkers may also help advance the application of PARPi in these settings $(78,79)$.

\section{Clinical evidence and studies}

\section{PARPi combination trials with radiotherapy}

The preclinical data led to the development of a phase I clinical trial (NCT03532880) to assess the safety of olaparib with cRT in SCLC patients. In this trial, ES-SCLC patients that have undergone $4-6$ cycles of a platinum-based agent and etoposide are being recruited. Patients will receive varying doses (ranging from 0 to $300 \mathrm{mg}$ ) of olaparib orally, twice daily, and cRT (30 Gy/10 fractions) similar to the Slotman et al. study (75). In addition, a similarly designed phase I study (NCT04170946) will examine this approach with talazoparib combined with the same cRT dose in ES-SCLC patients (summarized in Table 2). Of note, there are studies of olaparib in combination with RT in other histologies that are out of the scope of this review, but are listed here (NCT01562210, NCT02227082, 


\section{NCT02229656).}

\section{PARPi and immune oncology in SCLC}

Increasing evidence demonstrates the cGAS-STING pathway as a relevant mechanistic link between DNA damage and innate immune activation $(80,81)$. The activation of the cGAS-STING pathway leads to the recruitment and phosphorylation of TANK binding kinase 1 (TBK1) and interferon regulatory factor 3 (IRF-3), which ultimately activates the production of type I interferons (IFNs) (82). The secretion of IFNs, along with several other chemokines, promotes the recruitment of cytotoxic CD8+ T lymphocytes to tumor sites to effectively kill cancer cells (83).

Harding et al. demonstrated that DNA damage mediates innate immune activation in a cell cycle and cGAS-STING dependent manner. The inhibition of cell cycle progression or the impairment of the cGAS-STING pathway resulted in the loss of innate immune activity (80). Similarly, results from Mackenzie et al. highlighted the role that genomic instability plays in driving the formation of micronuclei following DNA damage where the cGAS molecule enters and triggers the subsequent downstream STING phosphorylation during the telophase of the cell cycle (81).

\section{Preclinical evidence and studies}

These findings prompted the investigation of novel approaches to harness DNA damage-induced innate immune activation for cancer therapy. Olaparib was subsequently reported by Sen et al. to activate the cGAS-STING pathway in SCLC, which enhanced the phosphorylation of TBK1 and IRF3 and ultimately stimulated the secretion of chemokines CCL5 and CXCL10. Olaparib also significantly increased the protein and surface expression levels of programmed-death ligand 1 (PD-L1) in SCLC models. The combination of olaparib and an anti-PD-L1 antibody was further evaluated in RPP immune-competent GEMMs. An evident increase in CD8+ cytotoxic $\mathrm{T}$ cell infiltration was accompanied by a decrease in tumor volume in these models (84).

\section{Clinical evidence and studies}

\section{PARPi combination trials with immunotherapy}

Despite these promising preclinical findings, a recent clinical trial (NCT02484404) by Thomas et al. found that the combination of olaparib and a PD-L1 inhibitor (durvalumab) had modest efficacy. In this trial, 20 relapsed ES-SCLC patients were treated with $300 \mathrm{mg}$ of olaparib twice a day and $1,500 \mathrm{mg}$ of durvalumab every four weeks. Only $2(10.5 \%)$ of the evaluable 19 patients had a complete or partial response to this treatment; the median PFS was 1.8 months. Grade 3 or 4 adverse effects were reported in $45 \%$ patients, with most being hematologic (61). In another phase II study (NCT02734004) that included 38 relapsed SCLC patients, Krebs et al. reported in abstract form that the combination of olaparib and durvalumab was well tolerated and 2 patients had confirmed partial or complete responses, however the primary endpoint of disease control rate (complete response, partial response and stable disease) at 12 weeks of $29 \%$ was in the futility region for this Bayesian designed study (62). An additional phase I trial (NCT02660034) that also includes relapsed SCLC is currently ongoing to assess the safety of PARP inhibitor pamiparib (BGB290) combined with anti-PD1 antibody tislelizumab (BGB-A317) (summarized in Table 2). Additional research is needed to optimize these novel immunotherapy-based combinations.

\section{Conclusions}

The prognosis for patients diagnosed with SCLC remains poor due to the aggressive nature and the frequent acquired resistance of this disease. The lack of effective and durable therapies contributes to its grim prognosis. Continual advances in our understanding of SCLC biology has shed light on targetable vulnerabilities, including PARP. Preclinical and growing clinical evidence suggest that PARPi can enhance treatment response in SCLC by acting as sensitizers of chemotherapy, targeted therapies, radiotherapy, and immunotherapy. Additionally, identifying biomarkers of SCLC treatment response to PARPi may optimize patient selection strategies. PARPi could be part of a future wave of novel therapeutics with the potential to impact SCLC patient outcomes.

\section{Acknowledgments}

Funding: The authors acknowledge support from Conquer Cancer the ASCO Foundation, the International Association for the Study of Lung Cancer, the Ontario Molecular Pathology Research Network of the Ontario Institute for Cancer Research, Lung Cancer Research Foundation, Canada Foundation for Innovation, Cancer 
Research Society, Canadian Institutes of Health Research (PJT169200), and the Clinical and Translational Science Center at Weill Cornell Medical Center and MSKCC (UL1TR00457; to BH Lok).

\section{Footnote}

Provenance and Peer review: This article was commissioned by the Guest Editor (Jose M. Pacheco) for the series "Small Cell Lung Cancer", published in Fournal of Thoracic Disease. This article has undergone external peer review.

Conflicts of Interest: All authors have completed the ICMJE uniform disclosure form (available at http://dx.doi. org/10.21037/jtd.2020.03.89). The series "Small Cell Lung Cancer" was commissioned by the editorial office without any funding or sponsorship. BHL reports grants from Pfizer, personal fees from AstraZeneca, outside the submitted work. The other authors have other conflicts of interest to declare.

Etbical Statement: The authors are accountable for all aspects of the work in ensuring that questions related to the accuracy or integrity of any part of the work are appropriately investigated and resolved.

Open Access Statement: This is an Open Access article distributed in accordance with the Creative Commons Attribution-NonCommercial-NoDerivs 4.0 International License (CC BY-NC-ND 4.0), which permits the noncommercial replication and distribution of the article with the strict proviso that no changes or edits are made and the original work is properly cited (including links to both the formal publication through the relevant DOI and the license). See: https://creativecommons.org/licenses/by-nc-nd/4.0/.

\section{References}

1. Cruz CSD, Tanoue LT, Matthay RA. Lung cancer: epidemiology, etiology, and prevention. Clin Chest Med 2011;32:605-44.

2. Bray F, Ferlay J, Soerjomataram I, et al. Global cancer statistics 2018: GLOBOCAN estimates of incidence and mortality worldwide for 36 cancers in 185 countries. CA Cancer J Clin 2018;68:394-424.

3. Govindan R, Page N, Morgensztern D, et al. Changing epidemiology of small-cell lung cancer in the United States over the last 30 years: analysis of the surveillance, epidemiologic, and end results database. J Clin Oncol 2006;24:4539-44.

4. GBD. Global, regional, and national age-sex-specific mortality for 282 causes of death in 195 countries and territories, 1980-2017: a systematic analysis for the Global Burden of Disease Study 2017. Lancet 2018;392:1736-88.

5. Helin K, Holm K, Niebuhr A, et al. Loss of the retinoblastoma protein-related $\mathrm{p} 130$ protein in small cell lung carcinoma. Proc Natl Acad Sci 1997;94:6933-8.

6. Kaye FJ. RB and cyclin dependent kinase pathways: defining a distinction between RB and p16 loss in lung cancer. Oncogene 2002;21:6908-14.

7. Ahrendt SA, Halachmi S, Chow JT, et al. Rapid p53 sequence analysis in primary lung cancer using an oligonucleotide probe array. Proc Natl Acad Sci 1999;96:7382-7.

8. Takahashi T, Takahashi T, Suzuki H, et al. The p53 gene is very frequently mutated in small-cell lung cancer with a distinct nucleotide substitution pattern. Oncogene 1991;6:1775-8.

9. D'amico D, Carbone D, Mitsudomi T, et al. High frequency of somatically acquired p53 mutations in small-cell lung cancer cell lines and tumors. Oncogene 1992;7:339-46.

10. George J, Lim JS, Jang SJ, et al. Comprehensive genomic profiles of small cell lung cancer. Nature 2015;524:47-53.

11. Rudin CM, Durinck S, Stawiski EW, et al. Comprehensive genomic analysis identifies SOX2 as a frequently amplified gene in small-cell lung cancer. Nat Genet 2012;44:1111-6.

12. Howlader N, Noone A, Krapcho M, et al. SEER Cancer Statistics Review, 1975-2016. National Cancer Institute. 2019. Available online: https://seer.cancer.gov/ csr/1975_2016/

13. FDA approves atezolizumab for extensive-stage small cell lung cancer. 2019. Available online: https://www.fda. gov/drugs/drug-approvals-and-databases/fda-approvesatezolizumab-extensive-stage-small-cell-lung-cancer

14. NCCN. Small cell lung cancer (version 2.2019). Available online: https://www.nccn.org/professionals/physician_gls/ pdf/sclc.pdf. Accessed April 12, 2019.

15. Sabari JK, Lok BH, Laird JH, et al. Unravelling the biology of SCLC: implications for therapy. Nat Rev Clin Oncol 2017;14:549.

16. Gazdar AF, Bunn PA, Minna JD. Small-cell lung cancer: what we know, what we need to know and the path forward. Nat Rev Cancer 2017;17:725.

17. Poirier JT, George J, Owonikoko TK, et al. New approaches to small cell lung cancer therapy: from the 
laboratory to the clinic. J Thorac Oncol 2020;S15560864:30056-3.

18. Satoh MS, Lindahl T. Role of poly(ADP-ribose) formation in DNA repair. Nature 1992;356:356-8.

19. Fong PC, Boss DS, Yap TA, et al. Inhibition of poly(ADPribose) polymerase in tumors from BRCA mutation carriers. N Engl J Med 2009;361:123-34.

20. Fong PC, Yap TA, Boss DS, et al. Poly(ADP)-ribose polymerase inhibition: frequent durable responses in BRCA carrier ovarian cancer correlating with platinumfree interval. J Clin Oncol 2010;28:2512-9.

21. Litton JK, Rugo HS, Ettl J, et al. Talazoparib in patients with advanced breast cancer and a germline BRCA mutation. N Engl J Med 2018;379:753-63.

22. Golan T, Hammel P, Reni M, et al. Maintenance olaparib for germline BRCA-mutated metastatic pancreatic cancer. N Engl J Med 2019;381:317-27.

23. Ledermann J, Harter P, Gourley C, et al. Olaparib maintenance therapy in platinum-sensitive relapsed ovarian cancer. N Engl J Med 2012;366:1382-92.

24. Moore K, Colombo N, Scambia G, et al. Maintenance olaparib in patients with newly diagnosed advanced ovarian cancer. N Engl J Med 2018;379:2495-505.

25. González-Martín A, Pothuri B, Vergote I, et al. Niraparib in patients with newly diagnosed advanced ovarian cancer. N Engl J Med 2019;381:2391-402.

26. Bryant HE, Schultz N, Thomas HD, et al. Specific killing of BRCA2-deficient tumours with inhibitors of poly (ADP-ribose) polymerase. Nature 2005;434:913-7.

27. Farmer H, McCabe N, Lord CJ, et al. Targeting the DNA repair defect in BRCA mutant cells as a therapeutic strategy. Nature 2005;434:917-21.

28. Murai J, Huang SY, Das BB, et al. Trapping of PARP1 and PARP2 by Clinical PARP Inhibitors. Cancer Res 2012;72:5588-99.

29. Murai J, Huang SY, Renaud A, et al. Stereospecific PARP trapping by BMN 673 and comparison with olaparib and rucaparib. Mol Cancer Ther 2014;13:433-43.

30. FDA. FDA approves olaparib for gBRCAm metastatic pancreatic adenocarcinoma. 2019. Available online: https://www.fda.gov/drugs/resources-informationapproved-drugs/fda-approves-olaparib-gbrcam-metastaticpancreatic-adenocarcinoma.

31. FDA. FDA approved olaparib (LYNPARZA, AstraZeneca Pharmaceuticals LP) for the maintenance treatment of adult patients with deleterious or suspected deleterious germline or somatic BRCA-mutated (gBRCAm or sBRCAm) advanced epithelial ovarian, fallopian tube or primary peritoneal cancer who are in complete or partial response to first-line platinum-based. 2018. Available online: https://www.fda.gov/drugs/fda-approved-olapariblynparza-astrazeneca-pharmaceuticals-lp-maintenancetreatment-adult-patients

32. FDA. FDA approves olaparib for germline BRCA-mutated metastatic breast cancer. 2018. Available online: https:// www.fda.gov/drugs/resources-information-approveddrugs/fda-approves-olaparib-germline-brca-mutatedmetastatic-breast-cancer

33. FDA. FDA approves rucaparib for maintenance treatment of recurrent ovarian, fallopian tube, or primary peritoneal cancer. 2018. Available online: https://www.fda.gov/drugs/ resources-information-approved-drugs/fda-approvesrucaparib-maintenance-treatment-recurrent-ovarianfallopian-tube-or-primary-peritoneal

34. FDA. FDA approves maintenance treatment for recurrent epithelial ovarian, fallopian tube or primary peritoneal cancers. 2017. Available online: https://www.fda.gov/newsevents/press-announcements/fda-approves-maintenancetreatment-recurrent-epithelial-ovarian-fallopian-tube-orprimary-peritoneal

35. FDA. FDA approves niraparib for HRD-positive advanced ovarian cancer 2019. Available online: https://www.fda. gov/drugs/resources-information-approved-drugs/fdaapproves-niraparib-hrd-positive-advanced-ovarian-cancer

36. FDA. FDA approves talazoparib for gBRCAm HER2negative locally advanced or metastatic breast cancer. 2018. Available online: https://www.fda.gov/drugs/drugapprovals-and-databases/fda-approves-talazoparibgbrcam-her2-negative-locally-advanced-or-metastaticbreast-cancer

37. FDA. FDA approves olaparib tablets for maintenance treatment in ovarian cancer. 2017. Available online: https:// www.fda.gov/drugs/resources-information-approveddrugs/fda-approves-olaparib-tablets-maintenancetreatment-ovarian-cancer

38. Pommier Y, O'Connor MJ, de Bono J. Laying a trap to kill cancer cells: PARP inhibitors and their mechanisms of action. Sci Transl Med 2016;8:362ps17.

39. Castroviejo-Bermejo M, Cruz C, Llop-Guevara A, et al. A RAD51 assay feasible in routine tumor samples calls PARP inhibitor response beyond BRCA mutation. EMBO Mol Med 2018. doi: 10.15252/emmm.201809172.

40. Abkevich V, Timms KM, Hennessy BT, et al. Patterns of genomic loss of heterozygosity predict homologous recombination repair defects in epithelial ovarian cancer. Br J Cancer 2012;107:1776-82. 
41. Birkbak NJ, Wang ZC, Kim JY, et al. Telomeric allelic imbalance indicates defective DNA repair and sensitivity to DNA-damaging agents. Cancer Discov 2012;2:366-75.

42. Popova T, Manie E, Rieunier G, et al. Ploidy and largescale genomic instability consistently identify basal-like breast carcinomas with BRCA1/2 inactivation. Cancer Res 2012;72:5454-62.

43. Watkins JA, Irshad S, Grigoriadis A, et al. Genomic scars as biomarkers of homologous recombination deficiency and drug response in breast and ovarian cancers. Breast Cancer Res 2014;16:211.

44. Telli ML, Jensen KC, Vinayak S, et al. Phase II Study of Gemcitabine, Carboplatin, and Iniparib As Neoadjuvant Therapy for Triple-Negative and BRCA1/2 MutationAssociated Breast Cancer With Assessment of a TumorBased Measure of Genomic Instability: PrECOG 0105.J Clin Oncol 2015;33:1895-901.

45. Isakoff SJ, Mayer EL, He L, et al. TBCRC009: A Multicenter Phase II Clinical Trial of Platinum Monotherapy With Biomarker Assessment in Metastatic Triple-Negative Breast Cancer. J Clin Oncol 2015;33:1902-9.

46. Byers LA, Wang J, Nilsson MB, et al. Proteomic profiling identifies dysregulated pathways in small cell lung cancer and novel therapeutic targets including PARP1. Cancer Discov 2012;2:798-811.

47. Polley E, Kunkel M, Evans D, et al. Small Cell Lung Cancer Screen of Oncology Drugs, Investigational Agents, and Gene and microRNA Expression. J Natl Cancer Inst $2016 ; 108$.

48. Murai J, Feng Y, Yu GK, et al. Resistance to PARP inhibitors by SLFN11 inactivation can be overcome by ATR inhibition. Oncotarget 2016;7:76534-50.

49. Lok BH, Gardner EE, Schneeberger VE, et al. PARP inhibitor activity correlates with SLFN11 expression and demonstrates synergy with temozolomide in small cell lung cancer. Clin Cancer Res 2017;23:523-35.

50. Stewart CA, Tong P, Cardnell RJ, et al. Dynamic variations in epithelial-to-mesenchymal transition (EMT), ATM, and SLFN11 govern response to PARP inhibitors and cisplatin in small cell lung cancer. Oncotarget 2017;8:28575.

51. Thomas A, Murai J, Pommier Y. The evolving landscape of predictive biomarkers of response to PARP inhibitors. J Clin Invest 2018;128:1727-30.

52. Mu Y, Lou J, Srivastava M, et al. SLFN11 inhibits checkpoint maintenance and homologous recombination repair. EMBO Rep 2016;17:94-109.

53. Murai J, Tang S-W, Leo E, et al. SLFN11 blocks stressed replication forks independently of ATR. Mol Cell 2018;69:371-84. e6.

54. Li M, Kao E, Malone D, et al. DNA damage-induced cell death relies on SLFN11-dependent cleavage of distinct type II tRNAs. Nat Struct Mol Biol 2018;25:1047-58.

55. de Bono J, Ramanathan RK, Mina L, et al. Phase I, dose-escalation, two-part trial of the PARP inhibitor talazoparib in patients with advanced germline BRCA1/2 mutations and selected sporadic cancers. Cancer Discov 2017;7:620-9.

56. Farago AF, Yeap BY, Stanzione M, et al. Combination Olaparib and Temozolomide in Relapsed Small-Cell Lung Cancer. Cancer Discov 2019;9:1372-87.

57. Hamilton E, Falchook GS, Wang JS, et al. Abstract CT025: Phase Ib study of adavosertib in combination with olaparib in patients with refractory solid tumors: Dose escalation. Cancer Research 2019;79:CT025.

58. Pietanza MC, Waqar SN, Krug LM, et al. Randomized, Double-Blind, Phase II Study of Temozolomide in Combination With Either Veliparib or Placebo in Patients With Relapsed-Sensitive or Refractory Small-Cell Lung Cancer. J Clin Oncol 2018;36:2386-94.

59. Owonikoko TK, Dahlberg SE, Sica GL, et al. Randomized Phase II Trial of Cisplatin and Etoposide in Combination With Veliparib or Placebo for Extensive-Stage Small-Cell Lung Cancer: ECOG-ACRIN 2511 Study. J Clin Oncol 2019;37:222-9.

60. Atrafi F, Groen HJM, Byers LA, et al. A Phase I DoseEscalation Study of Veliparib Combined with Carboplatin and Etoposide in Patients with Extensive-Stage Small Cell Lung Cancer and Other Solid Tumors. Clin Cancer Res 2019;25:496-505.

61. Thomas A, Vilimas R, Trindade C, et al. Durvalumab in Combination with Olaparib in Patients with Relapsed SCLC: Results from a Phase II Study. J Thorac Oncol 2019;14:1447-57.

62. Krebs M, Ross K, Kim S, et al. P1.15-004 An OpenLabel, Multitumor Phase II Basket Study of Olaparib and Durvalumab (MEDIOLA): Results in Patients with Relapsed SCLC. J Thorac Oncol 2017;12:S2044-5.

63. Lara Jr PN, Natale R, Crowley J, et al. Phase III trial of irinotecan/cisplatin compared with etoposide/cisplatin in extensive-stage small-cell lung cancer: clinical and pharmacogenomic results from SWOG S0124. J Clin Oncol 2009;27:2530.

64. Rossi A, Di Maio M, Chiodini P, et al. Carboplatinor cisplatin-based chemotherapy in first-line treatment of small-cell lung cancer: the COCIS meta-analysis of 
individual patient data. J Clin Oncol 2012;30:1692-8.

65. Paz-Ares L, Dvorkin M, Chen Y, et al. Durvalumab plus platinum-etoposide versus platinum-etoposide in firstline treatment of extensive-stage small-cell lung cancer (CASPIAN): a randomised, controlled, open-label, phase 3 trial. Lancet 2019;394:1929-39.

66. Horn L, Mansfield AS, Szczesna A, et al. First-Line Atezolizumab plus Chemotherapy in Extensive-Stage Small-Cell Lung Cancer. N Engl J Med 2018;379:2220-9.

67. Owonikoko TK, Zhang G, Deng X, et al. Poly (ADP) ribose polymerase enzyme inhibitor, veliparib, potentiates chemotherapy and radiation in vitro and in vivo in small cell lung cancer. Cancer Med 2014;3:1579-94.

68. Teicher BA, Silvers T, Selby M, et al. Small cell lung carcinoma cell line screen of etoposide/carboplatin plus a third agent. Cancer Med 2017;6:1952-64.

69. Lallo A, Frese KK, Morrow CJ, et al. The Combination of the PARP Inhibitor Olaparib and the WEE1 Inhibitor AZD1775 as a New Therapeutic Option for Small Cell Lung Cancer. Clin Cancer Res 2018;24:5153-64.

70. Gay CM, Tong P, Li L, et al. Abstract 2822: ATR inhibitors are active as single agents and in combination with PARP1 and ATM inhibitors in molecularly distinct subsets of small cell lung cancer models. Cancer Res 2018;78:2822.

71. Sen T, Tong P, Stewart CA, et al. CHK1 Inhibition in Small-Cell Lung Cancer Produces Single-Agent Activity in Biomarker-Defined Disease Subsets and Combination Activity with Cisplatin or Olaparib. Cancer Res 2017;77:3870-84.

72. Turrisi AT, 3rd, Kim K, Blum R, et al. Twice-daily compared with once-daily thoracic radiotherapy in limited small-cell lung cancer treated concurrently with cisplatin and etoposide. N Engl J Med 1999;340:265-71.

73. Faivre-Finn C, Snee M, Ashcroft L, et al. Concurrent once-daily versus twice-daily chemoradiotherapy in patients with limited-stage small-cell lung cancer (CONVERT): an open-label, phase 3, randomised, superiority trial. Lancet Oncol 2017;18:1116-25.

74. Jeremic B, Shibamoto Y, Nikolic N, et al. Role of radiation therapy in the combined-modality treatment of patients

Cite this article as: Barayan $\mathrm{R}$, Ran $\mathrm{X}$, Lok BH. PARP inhibitors for small cell lung cancer and their potential for integration into current treatment approaches. J Thorac Dis 2020;12(10):6240-6252. doi: 10.21037/jtd.2020.03.89 with extensive disease small-cell lung cancer: A randomized study. J Clin Oncol 1999;17:2092-9.

75. Slotman BJ, van Tinteren H, Praag JO, et al. Use of thoracic radiotherapy for extensive stage small-cell lung cancer: a phase 3 randomised controlled trial. Lancet 2015;385:36-42.

76. Gore EM, Hu C, Sun AY, et al. Randomized Phase II Study Comparing Prophylactic Cranial Irradiation Alone to Prophylactic Cranial Irradiation and Consolidative Extracranial Irradiation for Extensive-Disease Small Cell Lung Cancer (ED SCLC): NRG Oncology RTOG 0937. J Thorac Oncol 2017;12:1561-70.

77. Laird JH, Lok BH, Ma J, et al. Talazoparib Is a Potent Radiosensitizer in Small Cell Lung Cancer Cell Lines and Xenografts. Clin Cancer Res 2018;24:5143-52.

78. Carney B, Kossatz S, Lok BH. Target engagement imaging of PARP inhibitors in small-cell lung cancer. Nat Commun 2018;9:176.

79. Laird J, Lok BH, Carney B, et al. Positron-Emission Tomographic Imaging of a Fluorine 18-Radiolabeled Poly(ADP-Ribose) Polymerase 1 Inhibitor Monitors the Therapeutic Efficacy of Talazoparib in SCLC PatientDerived Xenografts. J Thorac Oncol 2019;14:1743-52.

80. Harding SM, Benci JL, Irianto J, et al. Mitotic progression following DNA damage enables pattern recognition within micronuclei. Nature 2017;548:466-70.

81. Mackenzie KJ, Carroll P, Martin CA, et al. cGAS surveillance of micronuclei links genome instability to innate immunity. Nature 2017;548:461-5.

82. Tanaka Y, Chen ZJ. STING specifies IRF3 phosphorylation by TBK1 in the cytosolic DNA signaling pathway. Sci Signal 2012;5:ra20.

83. Fuertes MB, Kacha AK, Kline J, et al. Host type I IFN signals are required for antitumor CD8+ T cell responses through CD8 $\{$ alpha $\}+$ dendritic cells. J Exp Med 2011;208:2005-16.

84. Sen T, Rodriguez BL, Chen L, et al. Targeting DNA Damage Response Promotes Antitumor Immunity through STING-Mediated T-cell Activation in Small Cell Lung Cancer. Cancer Discov 2019;9:646-61. 